\title{
Bound-based Spectrum Allocation for Cooperative Positioning
}

\author{
R. Raulefs*, S. Zhang, C. Mensing \\ German Aerospace Center (DLR), Germany
}

\begin{abstract}
This paper discusses the performance of distributed and centralized cooperative positioning system for multiple mobile terminals. A cooperative positioning system uses ranging between base stations and mobile terminals, and in addition exploits the links between the mobile terminals as well. However, the number of links in an all-to-all connected setup increases quadratically with the number of nodes. Our goal is to schedule the access for the available resources (used bandwidth) to track the nodes of a dynamic system. Therefore, it is essential to reason if the different links between the mobile terminals are useful. The reasoning assists in allocating resources properly. We investigate distributed algorithms that act based on the Cramér-Rao Lower Bound (CRLB). We use as benchmark a centralized and much more complex algorithm that requires all link information and we ignore any latency aspects. The results show that our distributed algorithm using limited information performs well. Copyright (c) 2012 John Wiley \& Sons, Ltd.
\end{abstract}

* Correspondence

Institute of Communications and Navigation, German Aerospace Center, 82234 Wessling, Germany

\section{INTRODUCTION}

Location information is of tremendous interest in wireless communication systems. Early 2000 Hightower et al. [1] described how ubiquitous location systems would support mobile applications. Mobile applications started to exploit location since mid of 2008 (basically after the iPhone $3 \mathrm{G}$ was released) as a survey of Skyhook showed [2]. Smartphones that offer location based applications obtain the location information using different technologies. The Global Positioning System (GPS) offers a solution that is well established since the beginning of 2000 e.g. many vehicular users apply GPS in their navigation systems to navigate their cars. However, indoors or in urban areas with high-raise buildings the direct access to the transmitted signal of the GPS satellites is blocked. The reception quality indoors of the GPS signals and the challenge of multi-path effects for the GPS receiver disable the successful use of GPS devices indoors [3, 4]. GPS receivers fail to operate in such environments and consequently these areas are defined as GPS-denied areas. Alternative solutions are in demand. Especially as the Federal Communications Commission (FCC) has restarted recently [5] the need to fulfill strict requirements to localize a mobile terminal by the cellular operator if the terminal calls 911. Mobile positioning indoors raises a lot of interest for numerous reasons.
Today terrestrial positioning for mobile terminals (MTs) uses only cellular base stations (BSs) as reference points [6]. This technique is also called non-cooperative positioning. Network operators plan their networks to fulfill the communication needs of each MT. At least one $\mathrm{BS}$ is required to establish and maintain a communication link. Therefore, the number of BSs with line of sight (LoS) condition may also be insufficient for trilateration positioning (e.g. up to three BSs per $1000 \mathrm{~m}^{2}$ ) [7]. As opposed to this in public indoor and urban scenarios the density of MTs is high (10-100 MTs per $\left.1000 \mathrm{~m}^{2}\right)$ [7]. Therefore, it is rather easy finding pairs of MTs that have LoS condition [8] which makes a precise ranging between MTs possible. Such pairs of MTs are under discussion as mobile relays in cellular mobile radio system [9]. These links are defined as peer-to-peer or device-to-device links. The main idea is that MTs additionally use the measurements of peer-to-peer links together with the neighboring MTs' position information to localize themselves cooperatively. Consequently this technique is called cooperative positioning [10],[11]. In 2000 Kurazume and Hirose [12] presented a cooperative positioning setup with numerous robots that cleaned the ground. In this setup the information was collected and processed at a central unit and presumed to be static between different measurements. Decentralized methodologies using short-range UWB devices have been investigated e.g. by Wymeersch et al. [13]. 
In this paper we discuss the potential to use the additional ranging information coming from the peer-topeer links gathered either at a centralized unit, such as the base station (or network), or locally inside the mobile terminal itself. Our focus is on the limitations that result from the number of possible links. Each link requires dedicated resources such as the available spectrum. For this we investigate several resource allocation methods that operate either locally or globally. The methods use as a basic criteria the Cramér-Rao Lower Bound (CRLB). The CRLB offers theoretically a good or a loose estimate of the performance $[14,15,16]$. The tightness of the bound depends on the available information. This is a key concern for a distributed system where only partial information is used. Therefore, we propose a novel combination of local resource allocation method together with a local approximation of the CRLB to apply cooperative positioning with numerous users simultaneously. For this the available spectrum for ranging is allocated to multiple terminals according to the requested demands of the mobile terminals. The CRLB is used as the decisive factor to decide which mobile terminal uses which spectrum band. We define this as a resource allocation problem [17] and compare different allocation schemes.

\section{SYSTEM MODEL}

\subsection{Notation of the System Model}

We setup our system model by defining the nodes in the network. Nodes in the network are either mobile terminals (MTs) or base stations (BSs).

Nodes: We consider a network with $K$ BSs $\left(B S^{1}, \ldots, B S^{K}\right)$ and $M$ MTs $\left(M T_{1}, \ldots, M T_{M}\right)^{*}$. We use 2-dimensional coordinate vectors to indicate the location of nodes:

$$
\begin{aligned}
\text { BS: } & \vec{r}^{k}=\left[x^{k}, y^{k}\right]^{T}, \quad \forall k \in(1, \ldots, K) \\
\text { MT: } & \vec{r}_{i}=\left[x_{i}, y_{i}\right]^{T}, \quad \forall i \in(1, \ldots, M) .
\end{aligned}
$$

The position estimations of the MTs are denoted as:

$$
\hat{\vec{r}}_{i}=\left[\hat{x}_{i}, \hat{y}_{i}\right]^{T}, \quad \forall i \in(1, \ldots, M) .
$$

We assume that the MTs and the BSs are always in range and can communicate with each other. Note: For better expression, in the remaining of the paper we also use $\chi^{k(i)}$ to denote the quantity $\chi$ referring to the $k^{\text {th }}$ neighboring BS of $M T_{i}$ and use $\chi_{j(i)}$ to denote the one referring to the $j^{\text {th }}$ neighboring MT of $M T_{i}$.

* In general, for better distinction we use superscript to indicate variables related to BS and subscript to MT.

\subsection{Ranging}

We mainly use TOA measurements for ranging. For simplicity we assume all the nodes are fully synchronized (i.e. there is no clock offset between nodes) ${ }^{\dagger}$. The signals propagate with the speed of light $(c=299792458 \mathrm{~m} / \mathrm{s})$. In general, the ranging measurement: $\rho[\mathrm{m}]$ is the measured propagation delay $\hat{\tau}[\mathrm{s}]$ multiplied by $c$ :

$$
\begin{aligned}
\rho & =\hat{\tau} \cdot c=(\tau+\varepsilon) \cdot c \\
& =\tau \cdot c+\varepsilon \cdot c=d+\eta,
\end{aligned}
$$

where $d$ is the true distance (known as the geometric line-of-sight (GLOS) distance), $\tau \triangleq d / c$ is the expected delay, $\varepsilon$ is the noise of the delay estimation and $\eta$ is the corresponding ranging error. From Equation (4) we can see for TOA the ranging error is proportional to the delay estimation error.

Terrestrial ranging For non-cooperative positioning, the MT ranges with its neighboring BSs, which we call terrestrial ranging. We assume the range of $B S^{k(i)}$ to $M T_{i}: \rho_{i}^{k(i)}$ is the true distance $d_{i}^{k(i)}$ with some noise $\eta_{i}^{k(i)}$ :

$$
\rho_{i}^{k(i)}=d_{i}^{k(i)}+\eta_{i}^{k(i)}
$$

Cooperative ranging For cooperative positioning, besides the terrestrial ranging, MTs also measure the distance from neighboring MTs via peer-to-peer links, called cooperative ranging:

$$
\rho_{i, j(i)}=d_{i, j(i)}+\eta_{i, j(i)},
$$

where $\rho_{i, j(i)}$ and $\eta_{i, j(i)}$ indicate the ranging and the measurement noise for the $M T_{j(i)} \rightarrow M T_{i}$ link (measured by $M T_{i}$ ) respectively. In the simplest setup, all measurement noise can be assumed as independent additive white Gaussian noise (AWGN) with an identical variance $\sigma^{2}$ for each dimension:

$$
\eta_{i}^{k(i)}, \eta_{i, j(i)} \sim \mathcal{N}\left(0, \sigma^{2}\right) .
$$

\subsection{Resources for Ranging}

There are several ways of how terminals could range between each other using a radio signal. Relevant parameters that influence the ranging performance are the used spectrum, the received signal power, the available time, the mobility of the terminals, hardware impairments, and potential changes in the environment. In our work we assume that the mobility of the mobile terminals is not significant. If multiple terminals are involved they may want to access the same resources such as the available spectrum for the radio link and the available time slot. Another resource could be the used transmit power of the radio signal to limit the spatial propagation of the radio

\footnotetext{
${ }^{\dagger}$ If the system is asynchronous, we can instead use RTD or TDOA.
} 
signal. Additionally to general aspects, also specifics of the applied communication scheme can be considered. Therefore, we investigate the optimal resource allocation scheme for a real OFDM system, where the noise variance normally depends on the link parameters, e.g. link distance (i.e. $d_{i}^{k}$ and $d_{i, j}$ ), occupied subcarriers, transmit power, shadowing, thermal noise, interference, etc.

\subsection{System and Problem Description}

The system contains BSs that are synchronized, always know their location information perfectly and share this information with MTs. In contrast to a MT that is not aware of its exact position. The goal of the system is to estimate MTs' positions as accurately as possible or with certain targeting accuracy: $\varrho_{i}[\mathrm{~m}]$. A MT collects other nodes' position information and measures the distance to make an estimate based on a positioning algorithm. In the mean time, MTs also offer their own position estimates to each other. In general, BSs are stationary whereas MTs move (reflected in our investigations by a mobility model). Sometimes a BS can also move and lose its position reliability (e.g. WiFi access point, femtocell base station, etc.). In this case it will be considered as a special MT and adjusts its own position information. Vice versa, when a MT has a very accurate location estimate, it also has the possibility to act as a BS, supporting others without being affected by the error propagation.

The system is modeled in a discrete time step sense to guarantee the fairness (i.e. all nodes are processing at the same time). Figure 1 shows a system flowchart. The system runs in several stages, which are: initialization, communications, resource allocation, movement, measurement and position estimate. In the initialization stage, each node is initialized based on a certain assumption, which could be deterministically located with a predefined position or statistically distributed following certain distributions. Based on the application, the initial estimate can be the true position, the noisy position information or random values. In the communications stage, each of the nodes broadcasts a message which contains its location information as well as some control signal. The control signal could be the reliability of its own location information, the resource it occupied, the environment of its neighborhood, etc. The resource allocation stage begins after nodes receive the messages from neighbors. Resources for measurement links will be allocated by the centralized coordinator or by the node itself based on its allocation scheme. In the moving stage, each node moves to a new position according to its mobility. In the measurement stage, each node performs range measurements. Then in a location estimate stage, a new position estimate can be obtained by combining the measurements and neighbors' position information which is read from the received messages. At the end, it goes back to the communications stage, where a node creates a new message with the new estimate and then broadcasts it again. The system moves to the next time step.

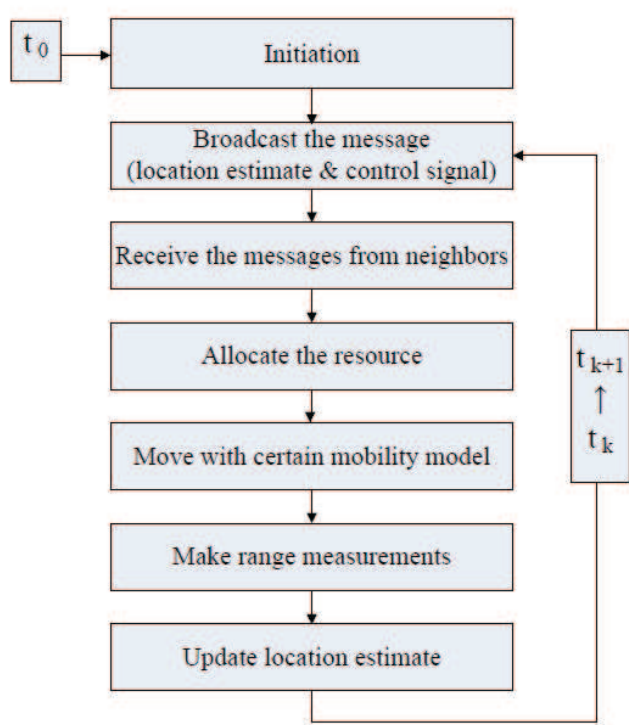

Figure 1. The flowchart of the system setup

\section{BOUNDS FOR COOPERATIVE POSITIONING}

In the following section we derive positioning bounds for a centralized system and an approximation of a bound that is used locally by each MT. The approach to apply the bounds offers the flexibility to smoothly transfer from a centralized to a distributed tracking method and vice versa. An example could be the particle filter. The bounds are later used by different resource allocation schemes to assess their potential to improve the performance. Let us presume there are in the strict sense two kinds of algorithms for tracking multiple objects at the same time. For centralized algorithms all measured data is forwarded to a single entity that processes the measured data. This works well if constraints like the coherence time of the used information is long enough and the objects have a direct link to the central unit. Distributed algorithms run on the objects itself (sometimes by sharing the computational load) to process measured and exchanged data that is shared between the objects by themselves.

\subsection{Cramér-Rao Lower Bound}

The Cramér-Rao Lower Bound (CRLB) expresses the lower bound of the variance of any estimation. In positioning it is applied to evaluate the performance of location estimators. We derive for non-cooperative and for cooperative positioning with independent ranging noise a centralized CRLB and for distributed systems a local approximation of the CRLB. The CRLB will serve in the next section as part of the utility function for the allocation schemes. 
For a parameter $\alpha$ estimated with the observation $g$, i.e. $\alpha \approx \hat{\alpha}(g)$, the CRLB theorem states :

$$
\operatorname{var}[\hat{\alpha}(g)] \geq \operatorname{CRLB}[\alpha]=\frac{1}{E\left[\left|\frac{d}{d \alpha} \ln p(g \mid \alpha)\right|^{2}\right]} .
$$

For multiple parameters $\alpha=\left[\alpha_{1}, \ldots, \alpha_{N}\right]^{T}$, the CRLB can be extended as a matrix:

$$
\operatorname{CRLB}[\alpha]=J[\alpha]^{-1},
$$

where $J[\alpha]$ is called Fischer Information Matrix (FIM). The entity of $J[\alpha]$ is defined as:

$$
J[\alpha]_{i, j}=-E\left[\frac{\partial^{2}}{\partial \alpha_{i} \partial \alpha_{j}} \ln p(g \mid \alpha)\right] .
$$

The variance of the parameter estimate is lower bounded by the diagonal element of CRLB matrix:

$$
\operatorname{var}\left[\hat{\alpha}_{i}\right] \geq \operatorname{CRLB}[\alpha]_{(i, i)} .
$$

\subsection{CRLB for Non-Cooperative Position Estimation}

In non-cooperative positioning, a MT only ranges with its neighboring BSs. We assume the terrestrial range $\rho_{i}^{k}$ is the distance measurement with location independent Gaussian noise:

$$
\rho_{i}^{k}=d_{i}^{k}+\eta_{i}^{k} \quad \eta_{i}^{k} \sim \mathcal{N}\left(0,\left(\sigma_{i}^{k}\right)^{2}\right),
$$

The FIM of $\vec{r}_{i}$ (in our case 2-dimensional, but it is in principle straightforward for 3-dimensional extension) is:

$$
\begin{aligned}
J_{\mathrm{nc}}\left[\vec{r}_{i}\right] & =-E\left[\frac{\partial^{2} \ln \left(p\left(\rho_{i}^{\mathrm{BS}} \mid \vec{r}_{i}\right)\right)}{\partial\left(\vec{r}_{i}\right)^{2}}\right] \\
& =H_{\mathrm{nc}, i}^{T} C_{\mathrm{nc}, i}^{-1} H_{\mathrm{nc}, i},
\end{aligned}
$$

With $H_{\mathrm{nc}, i}$ is the non-cooperative geometric matrix

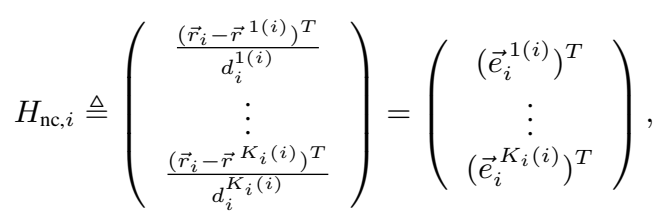

with $\vec{e}_{i}^{1(i)}$ is the unitary vector between the BS and the MT. $C_{\mathrm{nc}, i}$ is the non-cooperative ranging noise covariance matrix, which is diagonal when ranging errors from different links are uncorrelated:

$$
C_{\mathrm{nc}, i}=\left(\begin{array}{ccc}
\left(\sigma_{i}^{1(i)}\right)^{2} & \cdots & 0 \\
0 & \ddots & 0 \\
0 & \cdots & \left(\sigma_{i}^{K_{i}(i)}\right)^{2}
\end{array}\right)
$$

For an $M$ MTs non-cooperative positioning system, the FIM of the global parameter vector $\left(\vec{r}_{\mathrm{MT}}=\right.$ $\left.\left.\left[\begin{array}{lll}\left(\vec{r}_{i}\right)^{T} & \ldots & \left(\vec{r}_{M}\right.\end{array}\right)^{T}\right]^{T}\right)$ is a block diagonal matrix with the FIM of each MT along the diagonals:

$$
\begin{array}{r}
J_{\mathrm{nc}}\left[\vec{r}_{\mathrm{MT}}\right]=-E\left[\frac{\partial^{2} \ln \left(p\left(\rho_{\mathrm{MT}}^{\mathrm{BS}} \mid \vec{r}_{\mathrm{MT}}\right)\right)}{\partial\left(\vec{r}_{\mathrm{MT}}\right)^{2}}\right] \\
=\left(\begin{array}{ccc}
J_{\mathrm{nc}}\left[\vec{r}_{1}\right] & \cdots & 0 \\
0 & \ddots & 0 \\
0 & \cdots & J_{\mathrm{nc}}\left[\vec{r}_{M}\right]
\end{array}\right),
\end{array}
$$

where the $\rho_{\mathrm{MT}}^{\mathrm{BS}}$ denotes all the terrestrial ranges from all MTs.

\subsection{Centralized CRLB of Cooperative Position Estimation}

In non-cooperative positioning, the estimate of one MT is independent to the other MTs. Therefore, the global FIM has a block-diagonal structure. In cooperative positioning as shown before, besides the terrestrial ranging (BS-toMT), MTs also measure the distance from neighboring MTs via peer-to-peer links:

$$
\rho_{i, j}=d_{i, j}+\eta_{i, j} \quad \eta_{i, j} \sim \mathcal{N}\left(0,\left(\sigma_{i, j}\right)^{2}\right),
$$

where $\left(\sigma_{i, j}\right)^{2}$ is the measurement variance of $M T_{j} \rightarrow$ $M T_{i}$ link. With this cooperative ranging and the shared (estimated)location from neighbors, MTs are able to enhance their own positioning performance. However, due to the interaction between MTs' estimates, it is complicated to derive the CRLB for a single MT. In [16], Penna et al. derived a distributed cooperative positioning CRLB. They calculated the FIM for each user based on its own measurements' marginal likelihood function and then constructed the global FIM with all the local ones. In this subsection, we derive the centralized CRLB of cooperative positioning directly from the likelihood of the global measurements to find the theoretic lower bound of the estimator performance. We assume the ranging variance does not depend on location. Therefore, the FIM for the whole system can be formulated as:

$$
\begin{array}{r}
J\left[\vec{r}_{\mathrm{MT}}\right]=-E\left[\frac{\partial^{2} \ln \left(p\left(\rho_{\mathrm{MT}} \mid \vec{r}_{\mathrm{MT}}\right)\right)}{\partial\left(\vec{r}_{\mathrm{MT}}\right)^{2}}\right] \\
=J_{\mathrm{nc}}\left[\vec{r}_{\mathrm{MT}}\right]+J_{\mathrm{c}}\left[\vec{r}_{\mathrm{MT}}\right],
\end{array}
$$

with $J_{\mathrm{c}}\left[\vec{r}_{\mathrm{MT}}\right]$ is the cooperative part defined as:

$$
J_{\mathrm{c}}\left[\vec{r}_{\mathrm{MT}}\right] \triangleq \sum_{i=1}^{M} E\left[\frac{\partial^{2} \sum_{j \in \mathbb{M}_{i}} \frac{\left(d_{i, j}-\rho_{i, j}\right)^{2}}{2\left(\sigma_{i, j}\right)^{2}}}{\partial\left(\vec{r}_{\mathrm{MT}}\right)^{2}}\right] .
$$

Expanding (18), we can get:

$$
J_{\mathrm{c}}\left[\vec{r}_{\mathrm{MT}}\right] \triangleq\left(\begin{array}{ccc}
J_{\mathrm{c},(1,1)} & \cdots & J_{\mathrm{c},(1, M)} \\
\vdots & \ddots & \vdots \\
J_{\mathrm{c},(M, 1)} & \cdots & J_{\mathrm{c},(M, M)}
\end{array}\right),
$$

where $J_{\mathrm{c},(i, j)}$ is a $2 \times 2$ matrix $\forall i, j \in(1, \ldots, M)$. The block diagonal element $J_{\mathrm{c},(i, i)}$ can be rewritten as:

$$
J_{\mathrm{c},(i, i)}=H_{\mathrm{c}, i^{-}}^{T} C_{\mathrm{c}, \mathrm{bi}, i^{-}}^{-1} H_{\mathrm{c}, i^{-}}
$$


$C_{\mathrm{c}, \mathrm{bi}, i^{-}}^{-1}$ is the cooperative range weight matrix:

$$
C_{\mathrm{c}, \mathrm{bi}, i^{-}}^{-1}=\operatorname{diag}\left(c_{\mathrm{bi}, i, 1}^{-1}, \ldots, c_{\mathrm{bi}, i, j \neq i}^{-1}, \ldots, c_{\mathrm{bi}, i, M}^{-1}\right)
$$

$c_{\mathrm{bi}, i, j}^{-1} \triangleq \frac{\delta_{i, j}}{\left(\sigma_{i, j}\right)^{2}}+\frac{\delta_{j, i}}{\left(\sigma_{j, i}\right)^{2}}$ for simplicity, $\delta_{i, j}$ is the link selection factor:

$$
\delta_{i, j}= \begin{cases}1 & \text { if } M T_{j} \rightarrow M T_{i} \text { link is available; } \\ 0 & \text { if } M T_{j} \rightarrow M T_{i} \text { link is not available }\end{cases}
$$

and $H_{\mathrm{c}, i^{-}}$is the cooperative geometry matrix:

$$
H_{\mathrm{c}, i^{-}}=\left(\begin{array}{c}
\left(\vec{e}_{i, 1}\right)^{T} \\
\vdots \\
\left(\vec{e}_{i, j \neq i}\right)^{T} \\
\vdots \\
\left(\vec{e}_{i, M}\right)^{T}
\end{array}\right)
$$

Similarly, the non-block-diagonal elements $\left(\forall J_{c,(i, j)}\right.$ with $\left.i \neq j\right)$ are symmetric and can be derived as:

$$
J_{c,(i, j)}=J_{c,(j, i)}=-\vec{e}_{i, j} \cdot c_{\mathrm{bi}, i, j}^{-1} \cdot \vec{e}_{i, j}^{T} .
$$

We assume a MT is a neighbor only if both of the bidirectional links are available (mutual neighbor), i.e.:

$$
i \in \mathbb{M}_{j} \Leftrightarrow{ }^{\ddagger} j \in \mathbb{M}_{i}, \quad \forall i, j \in(1, \cdots, M) \text { and } i \neq j,
$$

This is reasonable for peer-to-peer links in mobile radio networks and necessarily to be true for a round trip delay (RTD) measurement between peers. Equation (20) can be simplified by:

$$
J_{\mathrm{c},(i, i)}=\tilde{H}_{\mathrm{c}, i}^{T} \tilde{C}_{\mathrm{c}, \mathrm{bi}, i}^{-1} \tilde{H}_{\mathrm{c}, i}
$$

where

$$
\left(\begin{array}{ccc}
\frac{\left(\sigma_{i, 1(i)}\right)^{2}+\left(\sigma_{1(i), i}\right)^{2}}{\left(\sigma_{i, 1(i)}\right)^{2} \cdot\left(\sigma_{1(i), i}\right)^{2}} & \cdots & \tilde{C}_{\mathrm{c}, \mathrm{bi} i}^{-1}= \\
0 & \ddots & 0 \\
0 & \cdots & \frac{\left(\sigma_{i, M_{i}(i)}\right)^{2}+\left(\sigma_{M_{i}(i), i}\right)^{2}}{\left(\sigma_{i, M_{i}(i)}\right)^{2} \cdot\left(\sigma_{M_{i}(i), i}\right)^{2}}
\end{array}\right),
$$

and

$$
\tilde{H}_{\mathrm{c}, i}=\left(\begin{array}{c}
\left(\vec{e}_{i, 1(i)}\right)^{T} \\
\vdots \\
\left(\vec{e}_{i, M_{i}(i)}\right)^{T}
\end{array}\right)
$$

\footnotetext{
$\ddagger \Leftrightarrow$ is read as 'is equivalent to'.
}

Equation (24) can be rewritten as:

$$
\begin{aligned}
& J_{c,(i, j)}=J_{c,(j, i)} \\
& = \begin{cases}-\vec{e}_{i, j} \cdot\left(\frac{1}{\left(\sigma_{i, j}\right)^{2}}+\frac{1}{\left(\sigma_{j, i}\right)^{2}}\right) \cdot\left(\vec{e}_{i, j}\right)^{T} & \text { if } j \in \mathbb{M}_{i} \\
0 & \text { if } j \notin \mathbb{M}_{i}\end{cases}
\end{aligned}
$$

The cooperative contribution can be obtained by inserting Equations (20), (24) (or (26), (29)) into Equation (19), and then we can get the overall FIM $J\left[\vec{r}_{\mathrm{MT}}\right]$ by Equation (17). By inverting $J\left[\vec{r}_{\mathrm{MT}}\right]$, we can have the global CRLB matrix for a cooperative positioning system:

$$
\mathrm{CRLB}_{\mathrm{c}}\left[\vec{r}_{\mathrm{MT}}\right]=J\left[\vec{r}_{\mathrm{MT}}\right]^{-1} .
$$

The variances of estimate coordinates with cooperation are lower bounded by the corresponding diagonal elements of $\left.\mathrm{CRLB}_{[} \vec{r}_{\mathrm{MT}}\right]$ :

$$
\operatorname{var}\left[\hat{\vec{r}}_{\mathrm{MT}, \mathrm{c}}(i)\right] \geq \mathrm{CRLB}\left[\vec{r}_{\mathrm{MT}}\right]_{(i, i)}, \quad \forall i \in(1, \ldots, M) .
$$

For bi-directional peer-to-peer links $\left(M T_{j} \rightarrow M T_{i}\right.$ and $\left.M T_{i} \rightarrow M T_{j}\right)$, the measurement variances are normally not identical:

$$
\left(\sigma_{i, j}\right)^{2} \nLeftarrow\left(\sigma_{j, i}\right)^{2},
$$

because of the different channel state information (CSI) and the adaptive resource allocation scheme. The CRLBs for non-cooperative and cooperative positioning as well as the simulated positioning estimation error are shown in Figure 2. We can see for non-cooperative positioning, the estimation error can achieve the CRLB and is quite stable. For cooperative positioning, the estimation accuracy is also close to the bound. The non-cooperative positioning performs better than the cooperative case because the bandwidth for each MT for the cooperative case is half of the non-cooperative, which makes it an unfair comparison.

\subsection{Approximate the Local Cooperative Positioning CRLB}

The previous derivations show for cooperative positioning the global FIM is normally not a block diagonal matrix. When calculating the global CRLB matrix, by the inverting operation, the entities interact with each other. That means the estimate error of one MT can directly affect the neighboring MTs who use this estimate as their reference. Furthermore, this error may even affect some non-neighboring MTs through some intermediate MTs. Therefore, for a dense network, calculating the positioning CRLB for a particular MT is difficult. On the other hand, for a distributed system there is no central unit collecting the global information. In this condition, how can a MT know its own estimate accuracy? Das and Wymeersch [18] proposed a scheme where each node calculates its own belief using the broadcasted beliefs of the neighbors.

Our approach approximates the lower bound of its own estimate variance only by local information. If the 


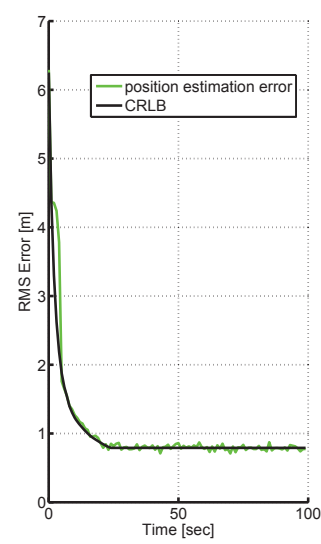

(a) non-cooper.

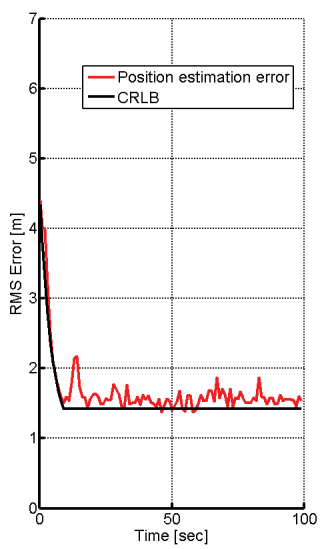

(b) cooper.
Figure 2. The positioning CRLB and the simulated positioning performance. For cooperative positioning we use two MTs that use the half of the bandwidth each. It is the average of 100 simulations.

neighbor's estimate variance is known we transfer the neighbor's location estimation inaccuracy to an equivalent ranging variance. For a peer-to-peer link $M T_{j} \rightarrow M T_{i}$, we assume the ranging measurement error (32) and the $M T_{j}$ 's estimation error in each dimension are normally distributed with known variances, i.e.:

$$
\begin{array}{ll}
\hat{x}_{j}=x_{j}+\epsilon_{j_{x}}, & \epsilon_{j_{x}} \sim \mathcal{N}\left(0,\left(\sigma_{j_{x}}\right)^{2}\right), \\
\hat{y}_{j}=y_{j}+\epsilon_{j_{y}}, & \epsilon_{j_{y}} \sim \mathcal{N}\left(0,\left(\sigma_{j_{y}}\right)^{2}\right) .
\end{array}
$$

We combine $M_{j}$ 's estimation variance with the ranging variance to get an equivalent ranging variance, i.e.:

$$
\left(\tilde{\sigma}_{i, j}\right)^{2}=f\left(\left(\sigma_{j_{x}}\right)^{2},\left(\sigma_{j_{y}}\right)^{2},\left(\sigma_{i, j}\right)^{2}\right) .
$$

With this transformation, we are able to calculate an equivalent non-cooperative CRLB just by replacing the true ranging variances by the equivalent ones. It is like assuming the neighbor's estimate is true, but the ranging measurement is less reliable. At the end of each time step, besides the location estimate, a MT also broadcasts its own local CRLB approximation so that others can use it as its position estimate variance to evaluate this link's quality. Sequentially, each MT can approximate its positioning CRLB and the extra communication effort is negligible (only one or two more values to share). It transfers the cooperative positioning problem into an equivalent noncooperative positioning one without losing the information of the estimations' inaccuracy of neighboring MTs. The equivalent ranging variance $\left(\tilde{\sigma}_{i, j}\right)^{2}$ can be derived as follow:

$$
\begin{aligned}
\rho_{i, j} & =\sqrt{\left(x_{i}-x_{j}\right)^{2}+\left(y_{i}-y_{j}\right)^{2}}+\eta_{i, j} \\
& =\sqrt{\left(x_{i}-\hat{x}_{j}+\epsilon_{j_{x}}\right)^{2}+\left(y_{i}-\hat{y}_{j}+\epsilon_{j_{y}}\right)^{2}}+\eta_{i, j},
\end{aligned}
$$

and

$$
\begin{array}{r}
\Delta \hat{x}_{i, j} \triangleq x_{i}-\hat{x}_{j}, \quad \Delta \hat{y}_{i, j} \triangleq y_{i}-\hat{y}_{j}, \\
\hat{d}_{i, j} \triangleq \sqrt{\left(x_{i}-\hat{x}_{j}\right)^{2}+\left(y_{i}-\hat{y}_{j}\right)^{2}} .
\end{array}
$$

The equivalent ranging variance states:

$$
\begin{aligned}
& \left(\tilde{\sigma}_{i, j}\right)^{2}=E\left[\left(\rho_{i, j}-\hat{d}_{i, j}\right)^{2}\right] \\
& =E\left[\left(\Delta \hat{x}_{i, j}+\epsilon_{j_{x}}\right)^{2}+\left(\Delta \hat{y}_{i, j}+\epsilon_{j_{y}}\right)^{2}+\hat{d}_{i, j}^{2}+\eta_{i, j}^{2}\right] \\
& +\underbrace{E\left[2 \eta_{i, j} \sqrt{\left(\Delta \hat{x}_{i, j}+\epsilon_{j_{x}}\right)^{2}+\left(\Delta \hat{y}_{i, j}+\epsilon_{j_{y}}\right)^{2}}\right]}_{=0} \\
& -\underbrace{E\left[2 \hat{d}_{i, j} \eta_{i, j}\right]}_{=0} \quad \underbrace{}_{\triangleq g\left(\epsilon_{j_{x}}, \epsilon_{j_{y}}\right)}[\underbrace{\left[\hat{d}_{i, j} \sqrt{\left(\Delta \hat{x}_{i, j}+\epsilon_{j_{x}}\right)^{2}+\left(\Delta \hat{y}_{i, j}+\epsilon_{j_{y}}\right)^{2}}\right.}] \\
& =\sigma_{j_{x}}^{2}+\sigma_{j_{y}}^{2}+\left(\sigma_{i, j}\right)^{2}+2 E\left[\hat{d}_{i, j}^{2}\right] \\
& +2 E\left[\Delta \hat{x}_{i, j} \epsilon_{j_{x}}\right]+2 E\left[\Delta \hat{y}_{i, j} \epsilon_{j_{y}}\right]-E\left[g\left(\epsilon_{j_{x}}, \epsilon_{j_{y}}\right)\right] .
\end{aligned}
$$

$g\left(\epsilon_{j_{x}}, \epsilon_{j_{y}}\right)$ can be polynomialized by taking the two variables Maclaurin expansion. If we approximate $g\left(\epsilon_{j_{x}}, \epsilon_{j_{y}}\right)$ with the second order expansion, the equivalent ranging variance becomes:

$$
\left(\tilde{\sigma}_{i, j}\right)^{2} \approx\left(\tilde{\sigma}_{i, j}\right)_{2^{\text {nd }}}^{2}
$$

and can be approximated as:

$$
\left(\tilde{\sigma}_{i, j}\right)^{2} \approx\left(\sigma_{i, j}\right)^{2}+\cos ^{2} \hat{\theta}_{i, j} \sigma_{j_{x}}^{2}+\sin ^{2} \hat{\theta}_{i, j} \sigma_{j_{y}}^{2},
$$

where $\hat{\theta}_{i, j} \triangleq$ angle $\left(\vec{r}_{i}-\hat{\vec{r}}_{j}\right)$. In a cooperative positioning system, $\hat{\theta}_{i, j}$ can be estimated by the old location estimate. Another way to estimate $\hat{\theta}_{i, j}$ is getting a rough location estimate at first.

In Figure 3 and 4 the (non-)cooperative positioning CRLB and the local cooperative CRLB approximation snapshots are compared. The standard deviation of all the links are set to be the same $(64 \mathrm{~m})$ and the neighbors' initial approximated CRLBs are set to infinity. We can see that the approximated CRLB is close to the true one. Also it is shown that the CRLB decreases when there are more cooperative nodes. Figure 5 shows the iterative approximation procedure averaging from 1000 simulations. It can be seen that the approximated local CRLB converges to a level which slightly higher than the true CRLB in a few iterations. From the simulation results we can see that the cooperative CRLB can be well approximated by the local approximation scheme we proposed. 


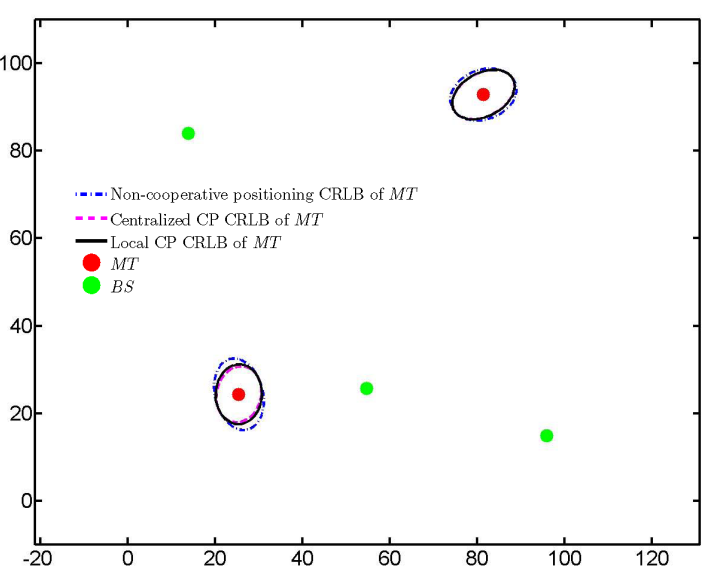

Figure 3. The comparison of the non-cooperative positioning CRLB (blue circle), the cooperative CRLB (magenta circle) and the local cooperative CRLB (black circle) approximation in 3 BSs (green dot) and 2 MTs (red dot) case. In this case the MT network is not dense, therefore, the cooperative gain is not significant. The three CRLBs are almost overlapping and difficult to distinguished.

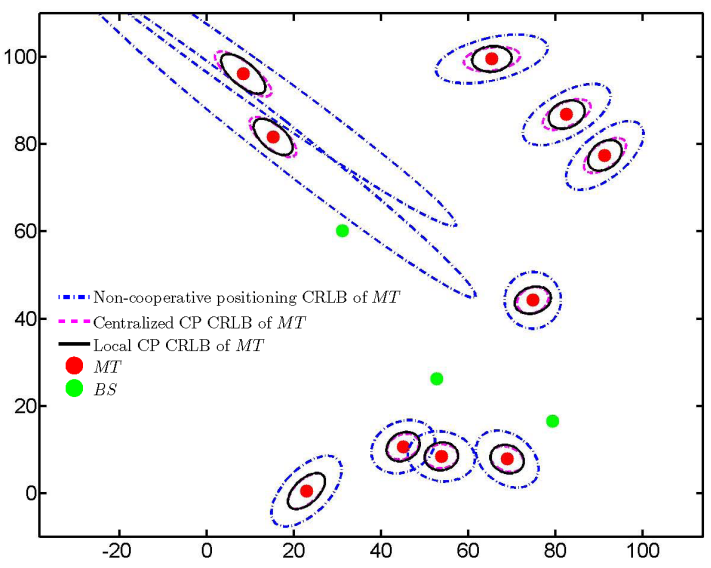

Figure 4. The comparison of the non-cooperative positioning CRLB (blue circle), the cooperative CRLB (magenta circle) and the local cooperative CRLB (black circle) approximation in 3 BSs (green dot) and 10 MTs (red dot) case. We can observe a significant gain through the cooperation. The local approximated CRLBs are almost overlapping with the true cooperative ones.

\section{RESOURCE ALLOCATION SCHEME FOR COOPERATIVE POSITIONING}

Most of the previous research in cooperative positioning assumes the ranging variances for all the links are identical or only depend on distance. Luise and Zanier described in [19] that the CRLB depends on multiple factors in a terrestrial positioning system. The authors showed that for a multicarrier signal, this variance also depends on the transmit power, carrier frequency, bandwidth, the number and indices of used subcarriers $\left(\left|S_{n}\right|^{2} \neq 0\right)$, etc.

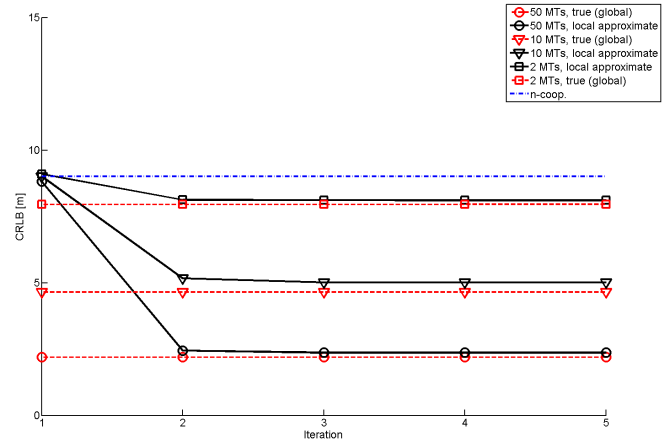

Figure 5. The comparison of the (non-) cooperative positioning CRLB and the local cooperative CRLB approximation

An OFDM signal is formulated as [19]:

$$
s(t)=\frac{1}{\sqrt{N}} \sum_{n=\left\lfloor-\frac{N-1}{2}\right\rfloor}^{\left\lfloor\frac{N-1}{2}\right\rfloor} S_{n} e^{j 2 \pi n f_{\mathrm{sc}} t}
$$

$f_{\text {sc }}$ is the subcarrier spacing, $N$ is the number of subcariers and $S_{n}$ is the information symbol carried by each subcarrier. Luise et al. derived the CRLB of the delay estimation in which states:

$$
\begin{aligned}
\operatorname{var}[\hat{\tau}] \geq \operatorname{CRLB}[\tau] & =\frac{1}{8 \pi^{2} f_{\mathrm{sc}}^{2} \sum_{n=\left\lfloor-\frac{N-1}{2}\right\rfloor}^{\left\lfloor\frac{N-1}{2}\right\rfloor} n^{2}\left|S_{n}\right|^{2} / \sigma_{0}^{2}} \\
& =\frac{1}{8 \pi^{2} f_{\mathrm{sc}}^{2} \sum_{n=\left\lfloor-\frac{N-1}{2}\right\rfloor}^{\left\lfloor\frac{N-1}{2}\right\rfloor} n^{2} \mathrm{SNR}_{n}}
\end{aligned}
$$

$\tau$ is the propagation delay, $\sigma_{0}^{2}$ is the variance of thermal noise.

The CRLB for ranging depicts that all the individual parameters, such as the subcarrier spacing $f_{S C}$, the number of subcarriers $N$, the index itself of the used subcarrier $n$, and the SNR of the used link can be understood as a resource that could be adapted to each link. Using different resources may lead to a different performance. The resource independent variance assumption from the previous research only holds when we consider a Time Division Multiple Access (TDMA) system, where all the links use the same spectrum resource within a specific time slot sequentially. In dense cooperative networks, the overall processing delay $\left(T_{\text {pro }}\right)$ of such a system is proportional to the total number of the links $L$ and increases quadratically with the number of MTs:

$$
T_{\text {pro }} \propto L \sim O\left(M^{2}\right) .
$$

In a static scenario, a high accuracy can be guaranteed because the delay will not cause any additional uncertainty. However, if a system is dynamic, MTs use the neighbors' 
old estimates as the references which are less reliable when the delay increases. To avoid this effect, we divide the spectrum resource into small parts to serve multiple links simultaneously (similar as the Frequency Division Multiple Access (FDMA) technique in communications). As already mentioned before, for a real wireless system, the resources are limited. In order to improve the overall performance, a resource optimization scheme is required. Because of the high diversity and the interaction due to the cooperation, it is difficult to get a global optimal solution. Alternatively, we can use some suboptimal approaches like the greedy algorithm. Moreover, recently the usage of game theory in wireless communication has been discussed, especially for a distributed system [20], [21]. Inspired by that, several resource allocation games are proposed for our distributed cooperative positioning system. Note we mainly consider the problem of allocating subcarriers in a multicarrier system. Although the precise definition of resource allocation may also include distributing power, time slot, etc.

In this section, a global greedy algorithm is introduced, which directly works with the global positioning CRLB. Then a partial decentralized approach inspired by the bidding game is raised to reduce the computational complexity. These two schemes will be compared with a centralized random allocation scheme (i.e. at each time step, certain amount of resources are allocated to a random link). At the end, we look into the purely decentralized case. First we introduce the non-cooperative game from the Nash equilibrium. Then we design a non-selfish utility function for our decentralized resource allocation game to reduce the interference.

\subsection{Centralized Greedy Allocation Scheme}

From the previous section, we obtained (or at least estimated) a global positioning CRLB matrix $(2 M \times$ $2 M)$ :

$$
\operatorname{CRLB}\left[\vec{r}_{\mathrm{MT}}\right]=\left(\begin{array}{ccc}
G_{(1,1)} & \cdots & G_{(1, M)} \\
\vdots & \ddots & \vdots \\
G_{(M, 1)} & \cdots & G_{(M, M)}
\end{array}\right)
$$

The trace of each block diagonal submatrix (Trace $\left[G_{(i, i)}\right]$ ) is the lower bound of the position variance in distance for each MT. The mean of these traces denotes the average performance of all the MTs, which refers to the system's efficiency. Whereas the variance of them shows the performance fluctuations of different MTs which measures the system's fairness. We focus our research on optimizing the efficiency.

The global CRLB matrix is obtained from the inverse of the global FIM, whose dimension increases with the number of MTs $(M)$. It is difficult to get a real global optimal solution when $M$ is high. Alternatively, the greedy algorithm is used which tries to optimize the sub-problems stepwise. Each time we take one piece of resources (could be a group of subcarriers), try to add them to each link, calculate a potential cost function and at the end assign it to the one with the lowest cost. Even with this scheme, the complexity is quite high. The computational complexity of allocating $N_{\text {res }}$ pieces of resource is $O\left(M^{5} N_{\text {res }}\right)$ (assuming the complexity of inverting a $N \times N$ matrix is $O\left(N^{3}\right)$ ), which will dramatically grow when the network density increases. The centralized approach requires a central unit with very high computation capacity.

\subsection{Partial Decentralized Allocation Bidding Scheme}

The resource allocation problem can also be analogically considered as a bidding scheme. A central resource pool contains all the free resources and works as a coordinator. Each candidate MT acts as a player of this scheme. At each time step, the resource pool chooses some resources and the players bid for it. The resource will be assigned to the player who offers the highest price. In our case, the price from each player is designed to denote how much improvement it will get with this these additional resources.

We derived an approximation of local positioning CRLB in Subsection 3.4. An MT $\left(M T_{i}\right)$ can add the potential new resources to each of its links and calculate the approximated local positioning CRLBs. The MT takes the smallest one and names it CRLB $\left[\vec{r}_{i}\right]_{\text {loc,new. The }}$ potential improvement can be obtained by subtracting CRLB $\left[\vec{r}_{i}\right]_{\text {loc,new }}$ from the current local CRLB approximation $\left(\mathrm{CRLB}\left[\vec{r}_{i}\right]_{\text {loc,cur }}\right)$. The value of this improvement are transmitted to the central resource pool as the bidding price of $M T_{i}$ :

$$
\text { Price }_{i}=\operatorname{CRLB}\left[\vec{r}_{i}\right]_{\mathrm{loc}, \mathrm{cur}}-\operatorname{CRLB}\left[\vec{r}_{i}\right]_{\mathrm{loc}, \text { new }} .
$$

The resource pool compares the prices from all the MTs and gives this resource to the one with the highest price. This resource will be used for this specific link.

The local CRLB matrix is the inverse of the local FIM (only $2 \times 2$ ). For a dense network the complexity of it can be neglected. Unlike the centralized greedy approach, for the bidding scheme the computations take place at both the MTs and the central unit. The MT's complexity linearly depends on the number of neighbors $(O)\left(M_{i}+\right.$ $\left.\left.K^{i}\right) N_{\text {res }}\right)$ ). The central unit's complexity depends on the number of MTs $\left(O\left(M N_{\text {res }}\right)\right)$. For dense network, the overall complexity is $O\left(M^{2} N_{\text {res }}\right)$.

\subsection{Non-Cooperative Allocation Game}

Game theory is a mathematical tool to analyze the rational behaviors of human in a competitive environment. It has been applied to predict politics and economy and to make decision in those areas. There are many types of game in the game theory. The most commonly used one is called the non-cooperative game. The idea of the non-cooperative game theory is as follow:

There are several players in a competitive game known as agents. The agents cannot communicate with 


\begin{tabular}{|c||c|c|c|c|}
\hline$a_{1}$ & null & $\mathrm{sc}_{1}$ & $\mathrm{sc}_{2}$ & $\mathrm{sc}_{1}, \mathrm{sc}_{2}$ \\
\hline \hline null & $(0,0)$ & $(0,1)$ & $(0,1)$ & $(0,2)$ \\
\hline $\mathrm{sc}_{1}$ & $(1,0)$ & $(0.1,0.1)$ & $(1,1)$ & $(0.1,1.1)$ \\
\hline $\mathrm{sc}_{2}$ & $(1,0)$ & $(1,1)$ & $(0.1,0.1)$ & $(0.1,1.1)$ \\
\hline $\mathrm{sc}_{1}, \mathrm{sc}_{2}$ & $(2,0)$ & $(1.1,0.1)$ & $(1.1,0.1)$ & $(0.2,0.2)$ \\
\hline
\end{tabular}

Table I. Non-cooperative resource allocation game

each other. An agent (say the $i^{\text {th }}$ one: $a_{i}$ ) has some candidate strategies $\lambda_{m(i)} \S_{i}, m=1,2, \ldots$, where $\Lambda_{i}$ is the strategies set for $a_{i}$. An utility function $u_{m(i)}$ can be formulated based on the strategies chosen by $a_{i}$ and the others which evaluates the benefit (can also be the cost, penalty, etc. depending on the type of the game) of this choice. A rational agent will be aware of others' potential strategies and the corresponding effects to itself. The goal of each agent is to optimize its utility function by applying a specific strategy. For a decentralized cooperative positioning system, MTs are considered as agents and the strategies set includes adding new subcarriers or not, which subcarriers to add, etc. First we investigate a simple case: Assuming two MTs are going to share two subcarriers $\left(\mathrm{sc}_{1}\right.$ and $\left.\mathrm{sc}_{2}\right)$. The strategies for each MT should contain not adding a new subcarrier (null), adding one subcarrier $\left(\mathrm{sc}_{1}\right.$ or $\left.\mathrm{sc}_{2}\right)$ and adding both $\left(\mathrm{sc}_{1}, \mathrm{sc}_{2}\right)$. We further assume if a subcarrier is used exclusively, it will contribute to the utility for one unit, but if a subcarrier is shared with both MTs, due to the interference, it only brings 0.1 unit contribution to each utility. A table of strategies and utility is presented in Table I. The only NE is obtained when both of the agents use both of the subcarriers. If there is no cooperative agreement, an agent will always behave selfishly and tries to occupy both of the subcarriers, even though $80 \%$ of the other joint allocation strategies achieve higher global utilities (except the three along the diagonal). The result can be extended to the scenario with more MTs and subcarriers. With non-cooperative resource allocation game, all MTs are trying to occupy as many subcarriers as possible. As the consequence, the interference will be dominant which may lead to a poor performance.

\subsection{Resource Allocation Game with Cooperative Behavior}

In the previous subsection, a non-cooperative allocation game was introduced, where the price of anarchy problem may be severe. To avoid this, an allocation game with the cooperative behavior is proposed. In this game, a MT is not a greedy agent anymore. It will be satisfied when its targeting estimation accuracy is achieved. Meanwhile, it evaluates the probability to have interference based on

\footnotetext{
$\S_{\text {}}$ This notation conflicts with the notation for the $M T_{i}$ 's $m^{\text {th }}$ neighboring MT and is used restrictively in this section.
}

the number of used subcarriers, and jointly chooses its resource allocation strategy.

We assume there are $N$ subcarriers and $M$ MTs. For the $i$ 'th terminal $M T_{i}$ the current number of subcarriers is defined as $n_{\mathrm{sc}, i}$, the targeting accuracy as $\varrho_{i}$ and the current local CRLB approximate as CRLB $\left[\vec{r}_{i}\right]_{\text {loc,cur. the relative }}$ need of improvement $\Delta_{i}$ is:

$$
\Delta_{i}=\frac{\operatorname{CRLB}_{\left[\vec{r}_{i}\right] \text { loc,cur }}-\left(\varrho_{i} \cdot \gamma\right)^{2}}{\varrho_{i}^{2}}
$$

$\gamma$ is the redundancy factor due to the fact that the CRLB may be not achievable by a suboptimal positioning algorithm. Instead of using a deterministic strategy, we use a statistic one. If $\Delta_{i}>0$ which means $M T_{i}$ is not satisfied with the current accuracy. We design $p_{i \text {,get }}$ as the probability of $M T_{i}$ getting some new subcarriers. $p_{i \text {,get }}$ depends on the required improvement and the guessed interference condition. Whereas if $\Delta_{i}<0$ which means the accuracy is more than enough. In order to reduce the potential interference for others, $M T_{i}$ is willing to release some subcarriers with the probability $p_{i, \text { release }}$.

Assuming all the other MTs have the same number of subcarriers randomly chosen from all the subcarriers. For $M T_{i}$, the probability that a single subcarrier is interfered by a specific neighbor $\left(M T_{j}\right)$ 's ranging signal is:

$$
p_{i, j, \text { one }}=\frac{\left(\begin{array}{c}
n_{\mathrm{sc}, i}-1 \\
N-1
\end{array}\right)}{\left(\begin{array}{c}
n_{\mathrm{sc}, i} \\
N
\end{array}\right)} \cdot p_{\text {intf }} .
$$

The probability that a single subcarrier is interfered reads:

$$
\begin{aligned}
p_{i, \text { one }} & =1-\left(1-\frac{\left(\begin{array}{c}
n_{\mathrm{sc}, i-1}-1 \\
N-1
\end{array}\right)}{\left(\begin{array}{c}
n_{\mathrm{sc}, i} \\
N
\end{array}\right)} \cdot p_{\text {intf }}\right)^{(M-1)} \\
& =1-\left(1-\frac{n_{\mathrm{sc}, i}}{N} \cdot p_{\text {intf }}\right)^{(M-1)}
\end{aligned}
$$

We define a percentage factor $\zeta \in[0,1]$. Then the probability that less than $\zeta n_{\mathrm{sc}, i}$ subcarriers get interference follows the Bernoulli distribution:

$$
p_{\zeta}=\sum_{k=0}^{\left\lceil n_{\mathrm{sc}, i} \cdot \zeta\right\rceil}\left(\begin{array}{c}
k \\
n_{\mathrm{sc}, i}
\end{array}\right) p_{i, \text { one }}^{k}\left(1-p_{i, \text { one }}\right)^{\left(n_{\mathrm{sc}, i}-k\right)} .
$$

If $\Delta_{i}>0$, probability of getting some new resources should be constructed in a way that it monotonically increases with respect to both $\Delta_{i}$ and $p_{\zeta}$. Moreover, it should not exceed the interval of $[0,1]$. We propose a function which fulfill the above constraints:

$$
p_{i, \mathrm{get}}=\nu_{\mathrm{dc}} \cdot e^{-\frac{1}{\Delta_{i}}}+\left(1-\nu_{\mathrm{dc}}\right) p_{\zeta},
$$

where $\nu_{\mathrm{dc}} \in[0,1]$ is a control factor to control the tradeoff between accuracy improvement and interference avoidance. If $\Delta_{i}<0$, the probability of releasing some occupied resources should be designed in a way that it monotonically decreases with respect of both $\Delta_{i}$ and $p_{\zeta}$. It should also be constrained by the interval $[0,1]$. Similarly, 

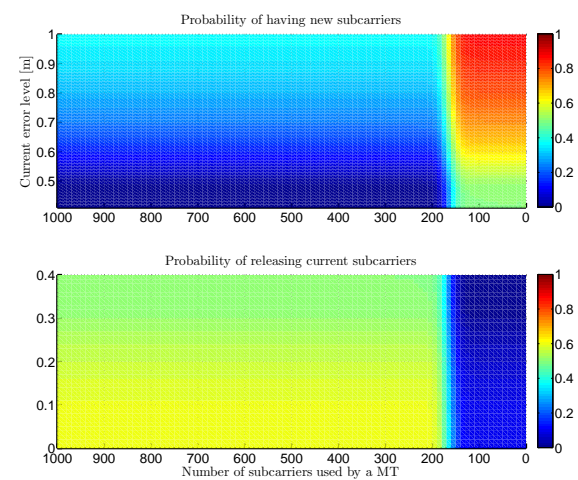

Figure 6. The probability of getting some new subcarriers (upper) and the probability of releasing some current subcarriers (lower). $M=5, N=1000, \varrho_{i}=0.5 \mathrm{~m}, p_{\text {intf }}=1, \zeta=0.5$ and $\gamma=0.8$

we design the function as follow:

$$
p_{i, \text { release }}=\nu_{\mathrm{dc}} \cdot e^{\frac{1}{\Delta_{i}}}+\left(1-\nu_{\mathrm{dc}}\right)\left(1-p_{\zeta}\right) .
$$

By this strategy, the overall number of used subcarriers is controlled by both current accuracy and chance of interference, which on some levels improves the performance. Figure 6 shows how the probability of getting or releasing subcarriers depends on the current local CRLB approximation and the number of subcarriers a MT already has.

\section{SIMULATION RESULTS}

In our simulations we differ between two scenarios. The first scenario differs between a close-by and far-away third base station. The two MTs build up the peer-topeer link and the resources are allocated depending on the distance to the far-away BS. The second scenario focuses on interference coordination and how interference limits the performance in case it is not well coordinated.

\subsection{Near- and Far-Choice (Scenario 1)}

In Scenario 1, we use the deterministic maps to compare the resource allocation solutions from different allocation schemes. three BSs and two MTs are located in the maps. We change the location of one BS to see the impact to the allocation solution (Figure 7 and 10). We used an OFDM system for positioning which has the total bandwidth of 20 $\mathrm{MHz}(0 \mathrm{MHz} \sim 20 \mathrm{MHz})$. The subcarrier spacing is set to 10 $\mathrm{KHz}$ and the carrier frequency is set to $5.2 \mathrm{GHz}$. Each used subcarrier transmits the signal with the power of $-30 \mathrm{dBm}$. Only TOA measurements are considered. The allocation solutions are shown in Figure 8, 9, 11 and 12.

From the simulation results we can see, if the BS is close by (Figure 7), the MTs will use the BS-MT links instead of the peer-to-peer links (Figure 8 and 9). Here, the

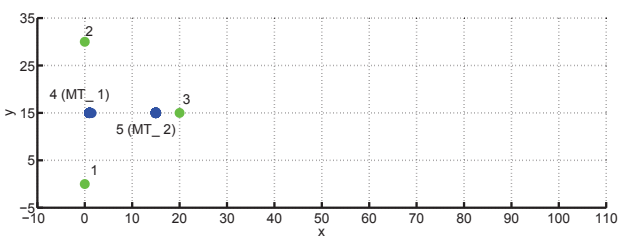

Figure 7. The map setup of Scenario 1. The green dots are the true positions of BSs and the blue ones are the MTs. In this case, the third BS is close to the MTs.

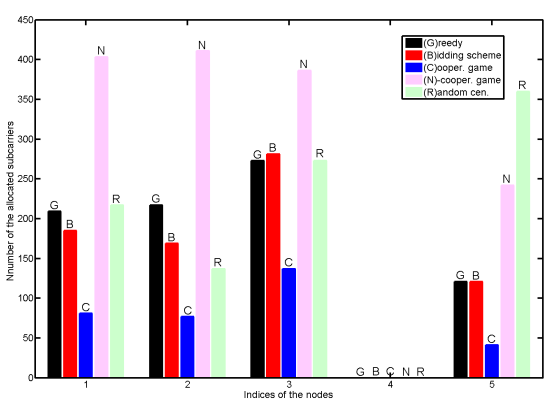

Figure 8. Comparison of the resource allocation solutions for $M T_{1}$ from different resource allocation schemes in Scenario 1 when the third BS is nearby.

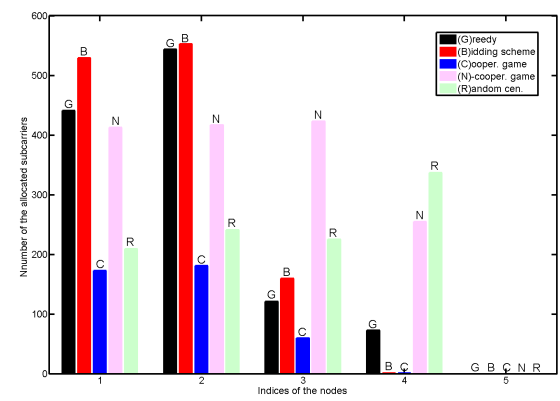

Figure 9. Comparison of the resource allocation solutions for $M T_{2}$ from different resource allocation schemes in Scenario 1 when the third BS is nearby.

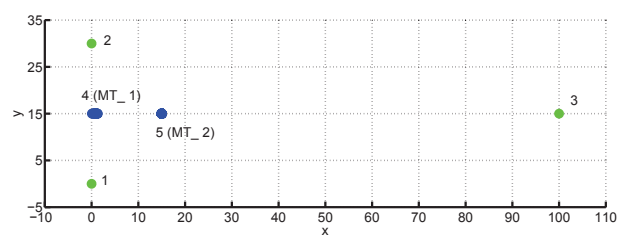

Figure 10. The map setup of scenario 1. The green dots are the true positions of BSs and the blue ones are the MTs. In this case, the third BS is far from the MTs.

location inaccuracy of the neighboring MT is considered. Whereas when a BS is far away (Figure 10), the MTs will 


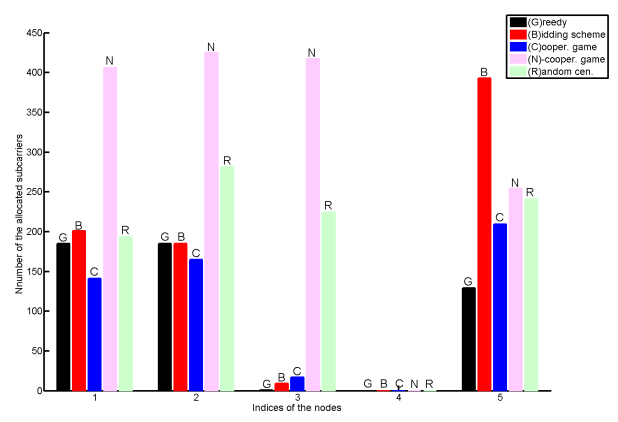

Figure 11. Comparison of the resource allocation solutions for $M T_{1}$ from different resource allocation schemes in Scenario 1 when the third BS is far away.

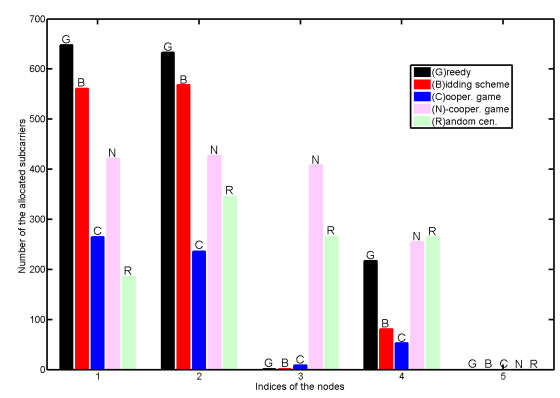

Figure 12. Comparison of the resource allocation solutions for $M T_{2}$ from different resource allocation schemes in Scenario 1 when the third BS is far away.

focus on the peer-to-peer links instead of the long distance BS-MT link (Figure 11 and 12). The SNR is attenuated due to the long propagation distance which decreases also the measurement reliability - see equation (41). Moreover, when a BS is far away, we can observe that the $M T_{1}$ put more effort on the peer-to-peer link than the $M T_{2}$ because of the geometric constellation. For $M T_{1}$, a reference point on the right can significantly decease the estimation uncertainty at $x$ dimension. Whereas for $M T_{2}$, an additional MT on the left will not offer significant gains because there have been already two BSs on the left. In general, we can find that the three allocation schemes we proposed make similar allocating decisions.

\subsection{Coordination of Resource (Scenario 2)}

In Scenario 2, thirteen BSs are located uniformly in a 60 $\mathrm{m} \times 60 \mathrm{~m}$ map, two MTs are located at the predefined positions. All the BSs and the MTs are stationary. We used a similar OFDM system as in Scenario 1, expect the total bandwidth is $2 \mathrm{MHz}(0 \mathrm{MHz}-2 \mathrm{MHz})$. We use GaussNewton for this scenario. The map setup can be found in Figure 13 and the simulation results (RMS (root mean square) error) with different resource allocation schemes are shown in Figure 14.

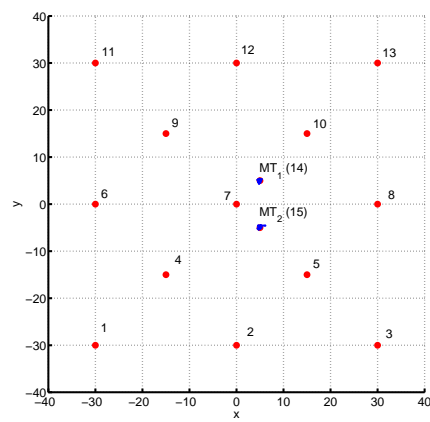

Figure 13. The map setup of scenario 2. The red dots are the true positions for both the BSs and the MTs. The blue traces are the connected estimations for MTs

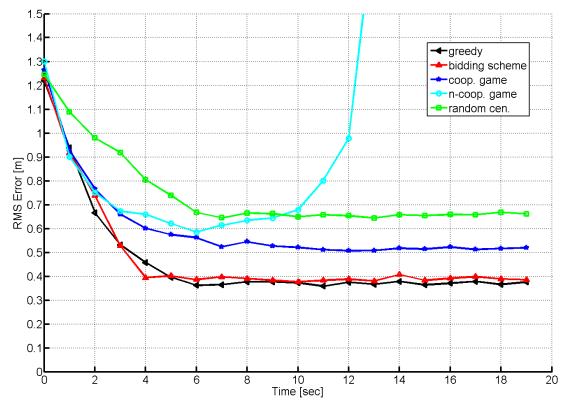

Figure 14. The comparison of different resource allocation schemes in Scenario 2

From Figure 14, we can see that in this scenario, the resource allocation schemes works well. The result of bidding game is close to the centralized greedy algorithm. However, the greedy algorithm has a much higher complexity: $O\left(M^{5} N_{\text {res }}\right)$ (Section 4.1), whereas the bidding game has a relatively low complexity: $O\left(M N_{\text {res }}\right)$ for the central unit and $O\left(M N_{\text {res }}\right)$ for each MT. Therefore, we can conclude that the bidding game is more suitable. The cooperative game performs worse than the greedy scheme and the bidding game but better than the random centralized approach and the non-cooperative game. Furthermore, we applied a decentralized approach that means the nodes do not know from each other which resources (subcarrier) the other nodes are using. This is taken into account by using the interference in the SINR - we replace the SNR in equation (41) with the SINR. For a decentralized approach the performance of the cooperative game is good. For the non-cooperative game, each MT always tries to increase its number of subcarriers. Therefore, after ten iterations, the interference is dominant which results in a rapidly increasing error. 


\section{CONCLUSIONS}

In this paper we presented two CRLB for cooperative positioning. The centralized CRLB was derived, and the local CRLB was approximated. The bounds were used as a parameter to assess the potential performance by using different resources of the peer-to-peer links between MTs. This information was applied for different resource allocation schemes. For a centralized system, a greedy algorithm is introduced which divides the problem into many sub-problems and tries to find the optimum for each sub-problem. The complexity of the greedy algorithm is high $O\left(M^{5} N_{\text {res }}\right)$. To reduce the complexity, a partial decentralized allocation scheme - bidding game is presented. The local approximated CRLB instead of the global CRLB is used to calculate the potential improvement for an MT. The simulation results show that the solution from bidding game is similar as the one from the greedy algorithm. The overall complexity is reduced to $O\left(M^{2} N_{\text {res }}\right)$. For a purely decentralized system, The resource allocation can be considered as a non-cooperative game. We evaluate the Nash equilibrium point where every MT behaves greedy and selfish. The interference becomes severe and jeopardizes the positioning estimation. To solve this problem, a cooperative game is presented. As a probabilistic approach, we design the utility function which fulfills the constraints. The simulation results show it works well in both stationary and dynamic cases. Due to the interference avoidance, the centralized schemes should in general outperforms the decentralized ones. However, from the simulation results we found out that sometimes the cooperative game performs better than the centralized greedy scheme. A reason for this could be the gain from reusing subcarriers is higher than the loss due to the interference.

\section{Acknowledgements}

This work has been performed in the project WHERE2 (Grant Agreement number 248894) and the COST Action IC0902, which are partly funded by the European Union under the European Communitys Seventh Framework Programme (FP7/2007-2013). The authors would like to thank the anonymous reviewers for their valuable comments and suggestions to improve the quality of the paper.

\section{REFERENCES}

1. J. Hightower and G. Borriello, "Location systems for ubiquitous computing," Computer, vol. 34, no. 8, pp. 57-66, 2001.

2. Skyhook, "Location apps research," 2011, http://www.skyhookwireless.com/locationapps/.

3. K. Pahlavan and F. Akgul and Y. Ye and T. Morgan and F. A.-Shabdiz and M. Heidari and C. Steger,
"Taking Positioning Indoors: Wi-Fi Localization and GNSS," InsideGNSS, vol. 5, no. 3, May 2010.

4. N. Patwari, J. Ash, S. Kyperountas, I. Hero, A.O., R. Moses, and N. Correal, "Locating the nodes: cooperative localization in wireless sensor networks," IEEE Signal Processing Magazine, vol. 22, no. 4, pp. $54-69$, July 2005.

5. FCC, "Enhanced 9-1-1 - wireless services," 2012, http://transition.fcc.gov/pshs/services/911services/enhanced911/Welcome.html.

6. M. I. Simic and P. V. Pejovic, "A comparison of three methods to determine mobile station location in cellular communication systems," European Transactions on Telecommunications, vol. 20, no. 8, pp. 711-721, 2009. [Online]. Available: http://dx.doi.org/10.1002/ett.1333

7. B. Uguen, "Scenarios and Parameters," WHERE2 (FP7-ICT-2009-4), Deliverable D1.1, August 2011.

8. J. Figueiras and S. Frattasi, Mobile Positioning and Tracking: From Conventional to Cooperative Techniques. John Wiley \& Sons, 2010.

9. K. Doppler, M. Rinne, C. Wijting, C. Ribeiro, and K. Hugl, "Device-to-Device Communication as an Underlay to LTE-Advanced Networks," IEEE Communications Magazine, vol. 47, no. 12, pp. 42 -49, Dec. 2009.

10. N. Patwari, J. Ash, S. Kyperountas, I. Hero, A.O., R. Moses, and N. Correal, "Locating the Nodes: Cooperative Localization in Wireless Sensor Networks," IEEE Signal Processing Magazine, vol. 22, no. 4, pp. 54 - 69, July 2005.

11. R. Kurazume and S. Hirose, "An experimental study of a cooperative positioning system," Autonomous Robots, vol. 8, pp. 43-52, 2000, 10.1023/A:1008988801987. [Online]. Available: http://dx.doi.org/10.1023/A:1008988801987

12. Ryo Kurazume and Shigeo Hirose, "An Experimental Study of a Cooperative Positioning System," $A U$ TONOMOUS ROBOTS, vol. 8, January 2000.

13. H. Wymeersch, J. Lien, and M. Win, "Cooperative Localization in Wireless Networks," Proceedings of the IEEE, vol. 97, no. 2, pp. 427 -450, Feb. 2009.

14. Y. Shen, H. Wymeersch, and M. Win, "Fundamental Limits of Wideband Localization - Part II: Cooperative Networks," IEEE Transactions on Information Theory, vol. 56, no. 10, pp. 4981 -5000, Oct. 2010.

15. M. Laaraiedh, S. Avrillon, and B. Uguen, "Cramerrao lower bounds for nonhybrid and hybrid localisation techniques in wireless networks," European Transactions on Telecommunications, 2011. [Online]. Available: http://dx.doi.org/10.1002/ ett. 1530

16. F. Penna, M. Caceres, and H. Wymeersch, "CramérRao Bound for Hybrid GNSS-Terrestrial Cooperative Positioning," IEEE Communications Letters, vol. 14, no. 11, pp. 1005 -1007, November 2010. 
17. B. Da and C. C. Ko, "Dynamic resource allocation in relay-assisted OFDMA cellular system," Transactions on Emerging Telecommunications Technologies, vol. 23, no. 1, pp. 96-103, 2012. [Online]. Available: http://dx.doi.org/10.1002/ett. 1509

18. K. Das and H. Wymeersch, "Censoring for bayesian cooperative positioning in dense wireless networks," IEEE Journal on Selected Areas in Communications (JSAC), Special issue on Cooperative Networking Challenges and Applications, - 2012.

19. M. Luise and F. Zanier, "Multicarrier signals: A natural enabler for cognitive positioning systems," in Multi-Carrier Systems \& Solutions 2009, ser. Lecture Notes in Electrical Engineering, S. Plass, A. Dammann, S. Kaiser, and K. Fazel, Eds. Springer Netherlands, 2009, vol. 41, pp. 313. [Online]. Available: http://dx.doi.org/10.1007/ 978-90-481-2530-2_1

20. A. B. MacKenzie and L. A. DaSilva, Game Theory for Wireless Engineers (Synthesis Lectures on Communications). Morgan \& Claypool Publishers.

21. D. Schmidt, C. Shi, R. Berry, M. Honig, and W. Utschick, "Distributed Resource Allocation Schemes," IEEE Signal Processing Magazine, vol. 26, no. 5, pp. 53-63, September 2009. 